\title{
Japanese Pronoun Acquisition by L1 English Adults: Further Evidence from a Coreference Judgment Task ${ }^{*}$
}

\author{
Carlos L. Pimentel \\ Department of World Languages and Literatures, Western Michigan University, Kalamazoo, U.S.A.
}

\begin{abstract}
This paper presents the results of a study investigating L2 learners' interpretations of Japanese overt and empty pronouns in a coreference judgment task. The results show counterevidence to Kanno's (1997, 1998) claim that learners at an early stage of language study obey the Overt Pronoun Constraint (Montalbetti, 1984), Furthermore, it supports evidence by Masumoto (2008), Pimentel and Nakayama (2012a) showing that learners at the early levels of Japanese learning do not show a knowledge of the constraint, but that this knowledge is acquired by the time they reach an advanced level of study.
\end{abstract}

Index Terms - overt and empty pronouns, OPC, bound variable, kanojo, kare, L2 Japanese

\section{INTRODUCTION}

A major research question in the field of second language (L2) acquisition research has been to what degree do adult learners achieve native-like competence in a target language. This is of special interest when linguistic features of the first language (L1) differ from those of the second. Japanese differs from English in that it is a language that can have either an overt or empty ( pro) pronoun in the subject position of a finite clause. In Japanese, kanojo and kare

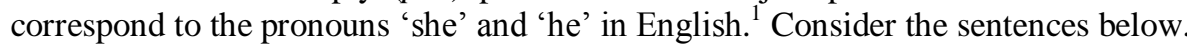

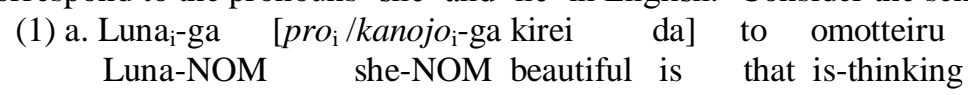

'Luna $\mathrm{i}_{\mathrm{i}}$ thinks that she $\mathrm{i}_{\mathrm{i}}$ is beautiful.'

b. Dono onna $\mathrm{i}_{\mathrm{i}}$-mo [pro $\mathrm{i}$ kirei da to omotteiru]

Every woman-too beautiful is that is-thinking

'Every woman ${ }_{\mathrm{i}}$ thinks that she $\mathrm{i}_{\mathrm{i}}$ is beautiful.'

c. *Dono onna $\mathrm{i}_{\mathrm{i}}$-mo $\left[\right.$ kanojo $_{i}$-ga kirei $\quad$ da] to omotteiru

Every woman-too she-NOM beautiful is that is-thinking

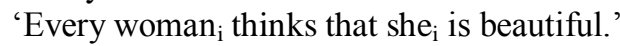

Sentence (1a) shows that regardless of whether the embedded subject pronoun is empty (pro) or overt (kanojo), it can take the referential NP (Luna) in the matrix clause as its antecedent. Sentences (1b) and (1c) contain a quantified NP antecedent in the matrix clause (i.e. dono onna-mo). Sentence (1b) shows that when the embedded subject is an empty pronoun (pro), it can take a quantified NP as its antecedent. Therefore, this particular interpretation where every woman thinks that she herself is beautiful is evinced, yields what is called a bound variable (BV) interpretation. On the other hand, sentence (1c) shows that when the embedded subject is an overt pronoun (kanojo), it cannot take a quantified NP as its antecedent, thereby making it ungrammatical. ${ }^{2}$ This restriction is known as the Overt Pronoun Constraint (OPC) (Montalbetti, 1984).

Japanese as a foreign language (hereafter JFL) learners whose first language (L1) is English are tasked with acquiring the relevant distinction between how overt pronouns with quantified NP antecedents function in the target language (i.e. Null Subject Parameter resetting). Sentential input containing a quantifier and an overt pronoun is infrequent and the constraint on BV interpretations is not explicitly taught in language classes (i.e. poverty of the stimulus argument). Thus, the question arises as to whether or not L1 English speakers learning Japanese can make the correct interpretations with respect to this phenomenon.

${ }^{*}$ The results in this paper are from my unpublished doctoral dissertation (Pimentel 2014), and were first presented with Mineharu Nakayama as a poster at the $14^{\text {th }}$ Annual International Meeting of the Japanese Society of Languages Sciences (JSLS) in Nagoya, Japan in 2012. An extended abstract of the results appeared in the handbook for the JSLS conference (Pimentel and Nakayama, 2012b).

${ }^{1}$ Hoji (1991) argues that kanozyo/kare are demonstratives rather than pronouns, but others (e.g. Kanno 1997, 1998) consider them to be pronouns. For the sake of consistency with Kanno $(1997,1998)$ and others, they will be referred to as pronouns in this paper. Regardless of nomenclature, kanozyo/kare exhibit similar distributional patterns to pronouns of other pro-drop languages (e.g. Spanish, Korean, Turkish, etc.)

${ }^{2}$ This follows Montalbetti's (1984) Overt Pronoun Constraint (OPC), which is formally defined as follows: "overt pronouns cannot link to formal variables iff the alternation overt/empty obtains." Note that the OPC becomes irrelevant if Japanese overt pronouns are indeed demonstratives. Since Kanno $(1997,1998)$ refer to the OPC, the author also mentions it in this paper. Even if the OPC it self is irrelevant theoretically, the fact that kare cannot have a quantifier antecedent remains. Thus, the main point does not change in this paper. 
A coreference judgment task conducted by Kanno $(1997,1998)$ gives evidence for JFL learners' ability to reset the Null Subject Parameter and thereby have knowledge of the OPC from a very early stage in their language learning. Masumoto (2008) and Pimentel and Nakayama (2012) provide counterevidence using a truth value judgment task. Their findings suggest that it takes time to reset the parameter, but that eventually by the advanced level of learning, JFL learners have acquired this knowledge.

This paper presents the experimental results of a coreferential judgment task designed to provide further inquiry. It focuses on L1 English speakers' grammatical judgments of sentences such as (1b) and (1c) above. In other words, it seeks to answer the following question: Do L1 English speakers acquire the knowledge that embedded overt pronouns cannot have main clause quantified NPs as their antecedent in Japanese?

The organization of this paper is as follows: section II presents the experiment, methodology, results, section III presents the discussion, and section IV presents the concluding remarks.

\section{EXPERIMENT}

The study compares five experimental groups: (i) a native control group; (ii) a group of $2^{\text {nd }}$ year L2 Japanese students; (iii) a group of $3^{\text {rd }}$ year L2 Japanese students; (iv) a group of $4^{\text {th }}$ year L2 Japanese students; (v) a group of $5^{\text {th }}$ year L2 Japanese students.

\section{A. Participants}

Forty-one native English speaking JFL learners (11 participants in $2^{\text {nd }}$ year Japanese (280 instructional hours), 12 in $3^{\text {rd }}$ year $(350 \mathrm{hrs}), 9$ in $4^{\text {th }}$ year $(550 \mathrm{hrs}), 9$ in $5^{\text {th }}$ year (beyond $\left.\left.600 \mathrm{hrs}\right)\right)$, and twenty Japanese native speakers (the control group) participated in the experiment. The participants were students taking Japanese language classes at a large midwestern university in the United States. The native speaker control group consisted of either graduate students at the same university or people who had studied at an American university for at least one year. All of the native speaker participants had finished their primary and secondary education in Japan and are fluent in English. Both the JFL learner group and the native speaker control group were recruited for participation by the experimenter either through direct means, which involved recruiting participants from their Japanese classes, or through e-mail solicitation. All of the participants were paid a nominal fee for their participation.

In Kanno's $(1997,1998)$ studies her participants were those who had 52 weeks of Japanese instruction. In the present study, the lowest level (Level 2) had approximately 280 hours of Japanese instruction, and was in the third quarter of their second year of Japanese study. As in Kanno's studies none of the Level 2 participants had ever lived in Japan or with a native speaker.

\section{B. Methodology}

The experiment employed a coreference judgment task in the form of a written questionnaire modeled after the one used in Kanno (1997, 1998). The participants were instructed to read the test sentences and answer a question that would determine their interpretation of the subject in the embedded clause. The answers were displayed in a multiplechoice format. The instructions clearly explained that the participants had the option of choosing answer (a), answer (b) or both (a) and (b) (hereafter referred to as answer (c)). The instructions were written in English and the test sentences were written in Japanese (hiragana, katakana and kanji). All of the vocabulary items were those that the students had previously studied. Furigana was listed over all of the words written in kanji to ensure that the students could read all of the sentences. The same questionnaire was given to both the JFL learners and the native speaker control group. The subjects were given the questionnaires to take home and complete at their leisure and then collected via written submission or e-mail at a later date.

The test sentences discussed in this paper comprised 4 types. Type 1: 3 sentences containing the quantified NP antecedent dare with an overt pronoun (kare/kanozyo). Type 2: 3 sentences containing the quantified NP antecedent dare with an empty pronoun. Type 3: 5 sentences containing the quantified NP antecedent dono $X$ mo with an overt pronoun (kare/kanozyo). Type 4: 5 sentences containing the quantified NP antecedent dono $X$ mo with an empty pronoun. The following are examples of the test sentences. The correct possible answers are in bold:

\section{(2) [Type 1] Overt pronoun with a quantified NP (dare) antecedent}

Dare-ga kinoo [kare-ga eewaziten-o $\quad$ kasita to itteiru $\mathrm{n}$ desu ka].

Who-NOM yesterday he-NOM English-Japanese dictionary-ACC lent that is saying that COP Q

'Who is saying that he lent the English-Japanese dictionary yesterday?'

Who-NOM yesterday English-Japanese dictionary-ACC lent that suppose Q

'Who do you suppose lent the English-Japanese dictionary yesterday?'
a) same as dare
b) another person
c) both (a) and (b)

(3) [Type 2] Empty pronoun with a quantified NP (dare) antecedent

Dare-ga kyoo [bideo-o miseru to] itteiru $\mathrm{n}$ desu ka].

Who-NOM today video-ACC show that is saying that COP Q

'Who said that (he) will show the video today?' 
Q: Dare-ga kyoo bideo-o miseru n desyoo ka.

Who-NOM today video-ACC show that suppose Q

Who do you suppose will show the video today?
a) same as dare
b) another person
c) both (a) and (b)

(4) [Type 3] Overt pronoun with a quantified NP (dono $X$ mo) antecedent

Dono ueetoresu-mo [kanozyo-ga Zyonson-san-ni denwa-suru to] itteimasu yo].

Which waitress-too she-NOM Johnson-DAT call-do that is saying

'Every waitress is saying that she will call Johnson.'

Q: Dare-ga denwa-suru $n$ desyoo ka.

Who-NOM call-do that suppose $\mathrm{Q}$

'Who do you suppose will call?'
a) same as dono ueetoresu
b) another person c) both (a) and (b)

(5) [Type 4] Empty pronoun with a quantified NP (dono $X$ mo) antecedent

Dono daigakusee-mo rainen [huransugo-o toru to] itteimasu yo].

Which college student-too next year French-ACC take that is saying

'Every college student is saying that next year (he) will take French.'

Q: Dare-ga huransugo-o toru $\mathrm{n}$ desyoo ka.

Who-Nom French-ACC take that suppose Q

'Who do you suppose will take French?'
a)same as dono daigakusee
b) another person
c) both (a) and (b)

Sentences of Types 1 and 3 contain a quantified NP antecedent (dare and dono X mo respectively) with an embedded overt pronoun (kare/kanozyo). Accordingly, the correct answer for these sentence types is (b). Sentences of Types 2 and 4 contain a quantified NP antecedent with an embedded empty pronoun, and accordingly, the correct answer for these sentence types can be either (a), (b) or (c). This is because these particular sentences are ambiguous in the sense that there are two possible interpretations: (i) coreferential reference and (ii) disjoint reference. Coreferential reference would be the interpretation where the empty pronoun takes the question word/quantifier as its antecedent. Disjoint reference would be the interpretation where the empty pronoun takes a sentence external antecedent.

\section{Results of QNP Sentences: Type 1}

Let us compare the results of the Type 1 sentences (dare with an overt pronoun) across all of the five JFL learner groups and the native speaker control group. The results are shown in Table I.

TABLE I.

RESULTS FOR QNP DARE OVERT (TYPE 1) SENTENCES

\begin{tabular}{cccc}
\hline Level & Answer (a) & Answer (b) & Answer (c) \\
\hline L2 $(n=11)$ & $21 / 33(63.6 \%)$ & $11 / 33(33.3 \%)$ & $1 / 33(3 \%)$ \\
L3 $(n=12)$ & $9 / 36(25 \%)$ & $26 / 36(72.2 \%)$ & $1 / 36(2.8 \%)$ \\
L4 $(n=9)$ & $3 / 27(11.1 \%)$ & $21 / 27(77.8 \%)$ & $3 / 27(11.1 \%)$ \\
L5 $(n=9)$ & $0 / 27(0 \%)$ & $26 / 27(96.3 \%)$ & $1 / 27(3.7 \%)$ \\
Natives $(n=20)$ & $0 / 60(0 \%)$ & $56 / 60(93.3 \%)$ & $4 / 60(6.7 \%)$ \\
\hline
\end{tabular}

In Levels 2 to 5, we see that Level 2 had the highest number of errors with $63.6 \%$ followed by a decrease in errors from Levels 3 to 5 . Level 3 made errors $25 \%$ of the time, followed by $11.1 \%$ of the time in Level 4 . The Level 5 group contained the lowest number of errors at $0 \%$ or at most $3.7 \%$ when (a) and (c) are combined. Comparatively, the number of correct rejections of the quantified NP (dare) as the antecedent of the overt pronoun was the lowest in Level 2 with 33.3\% followed by an increase in the higher levels with 72.2\% in Level 3, 77.8\% in Level 4, and 96.3\% in Level 5 . The native speaker control group made slightly more errors than the L5 group, however, they were comparable in that in both groups, no participants chose answer (a) only. However, the native speakers chose answer (c) higher (6.7\%) than the L5 learners (3.7\%). Although the native speakers did make some errors, their responses were still consistently accurate.

A one-way ANOVA reveals that the JFL and native speaker groups' (a) and (c) responses were significantly different $(\mathrm{F}(4,178)=16.705, \mathrm{p}<.001))$. A post-hoc Bonferroni test revealed a significant difference between the native speaker group and Level 2 ( $p<.001$ ). Level 2 also showed a significant difference with Levels 3 to 5 (all $p<.001$ ). No significant difference was found between the native speaker group and Level $3(\mathrm{p}=.069)$.

A one-way ANOVA of the Type 1 sentence percentages of students who chose only answer (a) showed a significant difference $(\mathrm{F}(4,178)=21.128, \mathrm{p}<.001)) .{ }^{3}$ A post-hoc Bonferroni test resulted in a significant difference between the native speaker group and each of Level 2 ( $p<.001)$, Level 3 ( $p<.05)$, and Level 4 ( $\mathrm{p}=.047)$. Significant differences were also found between the Level 2 group and each of Levels $3-5$ (all $p<.001$ ).

The percentage of individuals that consistently had incorrect ( 2 or more) responses by level. Incorrect responses were

${ }^{3}$ The total number of strictly (a) answers for Level 2 was $21(M=1.91, S D=1.04)$. The total number of strictly (a) answers for Level 3 was 9 ( $M=$ $0.75, S D=1.06)$. The total number of strictly (a) answers for Level 4 was $3(M=0.33, S D=0.71)$. The total number of strictly (a) answers for both Level 5 and the native speakers was 0. 
considered to be those responses other than (b) (i.e., choice (a) or (c)). These percentages are as follows: Level 2,8 participants (73\%), Level 3, 3 participants (25\%), Level 4, 2 participants (22\%), Level 5, 0 participants $(0 \%)$, Native participants, $0(0 \%)$. From these percentages, we can see that the Level 2 learners had the highest number of consistently incorrect responses at $73 \%$. The lowest percentage of consistently incorrect responses was found in Level 5 at $0 \%$. This percentage was the same as for the Native participants. The percentages show that the percentage of inconsistently incorrect answers decreases by level from Level 2 to 5.

D. Results of QNP Sentences: Type 3

Table II shows the percentages of the Type 3 sentences (dono X mo with an overt pronoun) across all of the levels.

TABLE II

RESULTS FOR QNP DONO OVERT (TYPE 3) SENTENCES

\begin{tabular}{cccc}
\hline Level & Answer (a) & Answer (b) & Answer (c) \\
\hline L2 $(\mathrm{n}=11)$ & $21 / 55(38.2 \%)$ & $26 / 55(47.3 \%)$ & $8 / 55(14.5 \%)$ \\
L3 $(\mathrm{n}=12)$ & $12 / 60(20 \%)$ & $35 / 60(58.3 \%)$ & $13 / 60(21.7 \%)$ \\
L4 $(\mathrm{n}=9)$ & $9 / 45(20 \%)$ & $30 / 45(66.7 \%)$ & $6 / 45(13.3 \%)$ \\
L5 $(\mathrm{n}=9)$ & $1 / 45(2.2 \%)$ & $39 / 45(86.7 \%)$ & $5 / 45(11.1 \%)$ \\
Natives $(\mathrm{n}=20)$ & $10 / 100(10 \%)$ & $84 / 100(84 \%)$ & $6 / 100(6 \%)$ \\
\hline
\end{tabular}

From the results, we can see that Level 2 had the highest number of errors with $38.2 \%$ and Level 5 had the lowest number of violations with $2.2 \%$. In fact, the Level 5 learners surpassed the native speaker control group in overall correct responses, but this was likely due to the fact that Level 5 had fewer participants (9) as compared to the native speakers (20). The Level 5 students also chose both answer (a) and (b) more (11.1\%) than the native speaker control group $(6 \%)$.

Interestingly, Level 3 and Level 4 had the same number of incorrect (a) responses at 20\%. However, the number of correct (b) responses was lower in Level $3(58.3 \%)$ than in Level 4 (66.7\%). The percentages for (c) responses was also higher in Level 3 than in Level 4 which shows that the Level 3 students were more likely to accept choice (a) as well as (b) as a possible correct answer more than the Level 4 participants. What we see in the results is a general trend for the Level 2 learners to treat the quantified NPs as viable antecedents for the overt pronouns in both the Type 1 and Type 3 sentences, and that as the level of the JFL learners increases, the number of correct rejections of the (a) answers decreases.

A one-way ANOVA reveals that the JFL and native speaker groups were significantly different in their (a) and (c) answers $(\mathrm{F}(4,300)=9.112, \mathrm{p}<.001)$. A post-hoc Bonferroni test showed that the native speaker group was significantly different in their (a) and (c) answers from the Level 2 and Level 3 groups ( $p<.001$ and $p<.05$, respectively). Both Levels 2 and 3 showed a significant difference from Level 5 ( $p<.001$ and $p<.05$, respectively).

Examining the strict answer (a) percentages for the Type 3 sentences resulted in a significant difference $(\mathrm{F}(4,300)=7.603, \mathrm{p}<.001)){ }^{4}$ A post-hoc Bonferroni test showed a significant difference between the native speaker group and Level 2 ( $\mathrm{p}<.001)$. The Level 2 and 3 groups showed no significant difference ( $\mathrm{p}=.079)$, but the Level 2 and 5 groups did show a significant difference $(\mathrm{p}<.001)$.

The percentage of participants that had consistently incorrect ( 3 or more) responses by level are as follows: Level 2 , 7 learners (64\%), Level 3, 6 learners (50\%), Level 4, 3 learners (33\%), Level 5, 1 learner (11\%), Native participants, 3 $(15 \%)$. In other words, seventeen out of fifty-one learners were consistently wrong. These results indicate that the Level 2 learners had the highest percentage of consistently incorrect responses (64\%) while the Level 5 learners had the lowest percentage of incorrect ones $(11 \%)$.

\section{E. Comparison of Type 1 and Type 3 Sentences}

Comparing the results from the Type 1 (QNP dare overt) and Type 3 (QNP dono overt) sentences, we see that for both sentence types the Level 2 participants made the most errors in comparison with Levels 3 to 5 . They also were significantly different in their responses from both the Level 5 participants and the native speaker control group. What is also observed is that as the level of the JFL learners increases, the number of errors decreases, which indicates that the Level 2 learners are utilizing a transfer strategy in their treatment of the overt pronouns, but learners at higher levels acquire the knowledge of the bound variable readings. This is in support of Masumoto and Nakayama (2009) and Pimentel and Nakayama's (2012a) claims that it takes time for JFL learners to learn that kare/kanozyo cannot take a bound variable reading.

A comparison of strictly (a) or (b) answers for each of the two sentence types shows that in Level 3, Level 4, and Level 5, the Type 1 sentences showed a higher percentage of correct (b) answers, but for the Level 2 group this percentage was higher in the Type 3 sentences. Similar to Level 3 to Level 5, the native speaker control group's responses revealed a higher percentage of correct (b) answers in the Type 1 sentences (93.3\% versus $84 \%$ in Type 3).

Comparing the individual responses, we see that the Level 2 learners had the highest percentage of errors with $73 \%$

${ }^{4}$ The total number of strictly (a) answers for Level 2 was $21(M=1.91, S D=1.22)$. The total number of strictly (a) answers for Level 3 was $12(M$ $=1.0, S D=1.41)$. The total number of strictly (a) answers for Level 4 was $10(M=1.0, S D=1.32)$. The total number of strictly (a) answers for Level 5 was $1(M=0.11, S D=0.33)$. The total number of strictly (a) answers for the native speakers was $10(M=0.5, S D=1.0)$. 
in both the Type 1 and Type 3 sentences. This was followed by a general decrease in the number of errors from Level 3 to Level 5. In the case of both sentence types, the Level 5 learners had the lowest percentage of consistently incorrect responses. Although the percentage of errors was higher for the Type 3 sentences for Levels 3 to 5 and the Native speaker group, this could have been due to the higher number of overall questions in the Type 3 sentences.

Eight out of the 51 JFL learners got two out of the three Type 1 sentences, and three out of the four Type 3 sentences wrong. In other words, $8 \mathrm{JFL}$ learners chose consistently incorrect answers for both the Type 1 and 3 sentences. Out of these eight learners, five were in in Level 2, two were in Level 3, and one was in Level 4. None of the Level 5 learners made consistently incorrect answers across both sentence types.

\section{F. Type 1 and Type 3 Sentences Combined}

Table III below shows the results of the Type 1 and 3 sentences combined.

TABLE III.

RESUlTS OF TYPE 1 AND 3 SENTENCES COMBINED

\begin{tabular}{cccc}
\hline Level & Answer (a) & Answer (b) & Answer (c) \\
\hline L2 $(n=11)$ & $42 / 88(47.7 \%)$ & $37 / 88(42.1 \%)$ & $9 / 88(10.2 \%)$ \\
L3 $(n=12)$ & $21 / 96(21.9 \%)$ & $61 / 96(63.5 \%)$ & $14 / 96(14.6 \%)$ \\
L4 $(n=9)$ & $12 / 72(16.7 \%)$ & $51 / 72(70.8 \%)$ & $9 / 72(12.5 \%)$ \\
L5 $(n=9)$ & $1 / 72(1.4 \%)$ & $65 / 72(90.3 \%)$ & $6 / 72(8.3 \%)$ \\
Natives $(n=20)$ & $10 / 160(6.25 \%)$ & $140 / 160(87.5 \%)$ & $10 / 160(6.25 \%)$ \\
\hline
\end{tabular}

The results show that the Level 2 learners made the most errors at $47.7 \%$ and accepted answer (c) $10.2 \%$ of the time. The Level 5 learners made the lowest number of errors at $1.4 \%$ choosing answer (a), $90.3 \%$ choosing answer (b) and $8.3 \%$ choosing answer (c). The native speaker control group made more errors than the Level 5 learners $(6.25 \%$ and $1.4 \%$ respectively for answer (a) only). The percentage of errors was higher in Level 3 (21.9\%) as compared with Level $4(16.7 \%)$. The combined percentages show that there was a decrease in the number of overall errors from Level 2 to Level 5.

Let us now look at the number of individuals who made consistent errors in both the Type 1 and Type 3 sentences. These numbers are as follows: Level 2, 5 learners (45\%), Level 3, 2 learners (17\%), Level 4, 1 learner (11\%), and Level $5,0 .^{5}$ These results show a decrease in the number of consistent errors by group indicating that over time and with exposure to Japanese, the learners are gaining knowledge that overt pronouns cannot be bound by quantified NP antecedents.

\section{G. Results of Type 2 Sentences}

Table IV lists the results for the Type 2 sentences. These sentences contained an empty pronoun with dare. In these sentences the participants had to decide whether the empty pronoun would be coreferential with the quantified NP antecedent dare or an extra-sentential referent. All the answers below are correct and show the preferences of the participants.

TABLE IV.

RESULTS OF QNP DARE EMPTY (TYPE 2) SENTENCES

\begin{tabular}{cccc}
\hline Level & Answer (a) & Answer (b) & Answer (c) \\
\hline L2 $(n=11)$ & $23 / 33(69.7 \%)$ & $8 / 33(24.2 \%)$ & $2 / 33(6.1 \%)$ \\
L3 $(n=12)$ & $28 / 36(77.8 \%)$ & $8 / 36(22.2 \%)$ & $0 / 36(0 \%)$ \\
L4 $(n=9)$ & $22 / 27(81.5 \%)$ & $2 / 27(7.4 \%)$ & $3 / 27(11.1 \%)$ \\
L5 $(n=9)$ & $12 / 27(44.5 \%)$ & $5 / 27(18.5 \%)$ & $10 / 27(37 \%)$ \\
Natives $(n=20)$ & $27 / 60(45 \%)$ & $22 / 60(36.7 \%)$ & $11 / 60(18.3 \%)$ \\
\hline
\end{tabular}

As per the results, we can see that all of the JFL learners and the native speaker control group showed a higher preference for the intra-sentential quantified NP antecedent dare to be the antecedent of the empty pronoun. Interestingly, the answer (a) responses rose from Level 2 to Level 4 (L2: 69.7\%, L3: 77.8\%, L4: 81.5\%). The percentage of answer (b) responses also decreased by level from Level 2 to Level 4 (L2: 24.2\%, L3: 22.2\%, L4: 7.4\%). The percentage of (c) responses was lowest in Level $3(0 \%)$ and highest in Level $5(37 \%)$. These results indicate that the JFL learners prefer the quantified NP to be the antecedent of kare/kanozyo. Comparing the Level 5 learners with the native speakers, we find that both groups showed higher percentages for answer (a) (44.5\% and $45 \%$ respectively), but that the native speaker group percentage for answer (b) was higher.

A one-way ANOVA showed the groups were significantly different in their (a) and (c) answers $(F(4,178)=2.481$, $\mathrm{p}<.05)$. A post-hoc Bonferroni test showed that the native speaker control group was significantly different from Level $4(\mathrm{p}<.05)$.

Now let us look at the number of participants that consistently chose (a) (two out of three times). The percentages by level are as follows: Level 2, 8 learners (73\%), Level 3, 10 learners (83\%), Level 4, 9 learners (100\%), Level 5, 3

\footnotetext{
${ }^{5}$ The sum of strictly (a) answers for the Type 1 plus the Type 3 sentences in Level 2 was $42(M=3.82, S D=1.78)$. For Level 3, the sum was 21 ( $M$ $=1.75, S D=1.86)$. For Level 4 , the sum was $12(M=1.33, S D=1.22)$. For Level 5 , the sum was $1(M=0.11, S D=0.33)$. For the native speakers, the sum was10 $(M=0.5, S D=1.0)$.
} 
learners (44\%), and Native participants, 9 (45\%). The percentages for participants that consistently chose (b) (two out of three times) by level are as follows: Level 2, 2 learners (18\%), Level 3, 2 learners (17\%), Level 4, 0 learners (0\%), Level 5, 1 learner (11\%), and Native participants, 8 (40\%). The percentages for participants that consistently chose (c) (two out of three times) by level are as follows: Level 2, 1 learner (9\%), Level 3, 0 learners (0\%), Level 4, 0 learners (0\%), Level 5, 3 learners (33\%), and Native participants, 3 (15\%).

These results showed that the JFL learners had a generally high preference for the QNP dare to be the antecedent of the empty pronoun. An increase was found in the percentage of (a) answers from Levels 2 to 4 . However, the Level 5 participants showed the lowest consistency for (a) answers (45\%) and the highest consistency for (c) answers (33\%). The Native speakers showed a higher consistency for (a) answers (40\%) than (b) answers (45\%) with (c) showing the least consistency (15\%) in this group. The higher percentage of consistently (a) answers for Levels 2 to 4 indicates a much higher preference for (a) answers among these groups.

\section{H. Results of Type 4 Sentences}

The results of the Type 4 sentences in Table V show that the JFL learners showed a higher percentage of (a) responses with comparison to (b) and (c) across the four groups. This means that the JFL learners showed a higher preference for the quantified NP dono X mo to be the antecedent of the empty pronoun in contrast with either an extrasentential antecedent (answer (b)) or both the quantified NP and an extra-sentential antecedent (answer (c)).

TABLE V.

RESULTS FOR QNP DONO X MO EMPTY (TYPE 4) SENTENCES

\begin{tabular}{cccc}
\hline Level & Answer (a) & Answer (b) & Answer (c) \\
\hline L2 $(n=11)$ & $34 / 55(61.8 \%)$ & $11 / 55(20 \%)$ & $10 / 55(18.2 \%)$ \\
L3 $(n=12)$ & $33 / 60(55 \%)$ & $3 / 60(5 \%)$ & $24 / 60(40 \%)$ \\
L4 $(n=9)$ & $29 / 45(64.4 \%)$ & $10 / 45(22.2 \%)$ & $6 / 45(13.4 \%)$ \\
L5 $(n=9)$ & $23 / 45(51.1 \%)$ & $4 / 45(8.9 \%)$ & $18 / 45(40 \%)$ \\
Natives $(n=20)$ & $90 / 100(90 \%)$ & $2 / 100(2 \%)$ & $8 / 100(8 \%)$ \\
\hline
\end{tabular}

In comparing answer (c) across the JFL learner groups, we see that the highest percentages for this choice were in Level 3 and Level 5 (40\% each), and the lower percentages were in Level 2 and Level 4 (18.2\% and 13.4\% respectively). The native speaker control group showed a strong preference for the quantified NP to be the antecedent of the empty pronoun (answer (a)) at 90\%. They only chose answer (b) and answer (c) $2 \%$ and $8 \%$ of the time, respectively. A one-way ANOVA revealed a significant difference in the (a) and (c) answers $(F(4,300)=6.043, p<.001)$ ). A post-hoc Bonferroni test showed that the native speaker group's responses were significantly different from the Level 2 and Level 4 groups ( $<<.05$ and $p<.001$, respectively). The Level 2 and Level 3 groups did not show a significant difference $(\mathrm{p}=.057)$, but the Level 3 and Level 4 groups did show a significant difference $(\mathrm{p}<.05)$.

The percentages for participants that consistently chose (a) are as follows: Level 2, 7 learners (64\%), Level 3, 8 learners (67\%), Level 4, 6 learners (67\%), Level 5, 6 learners (67\%), and Native participants, 19 (95\%). From this we can see that the percentage of learners that chose (a) consistently differed very little from Levels 2 to 5 . The percentages of learners that chose (b) consistently by level are as follows: Level 2, 1 learner (9\%), Level 3, 0, Level 4, 2 learners (22\%), Level 5, 0, and Native participants, 0 . The percentages of learners that chose (c) consistently by level are as follows: Level 2, 3 learners (27\%), Level 3, 4 learners (33\%), Level 4, 1 learner (11\%), Level 5, 3 learners (33\%), and Native participants, $1(5 \%)$.

For the percentages of participants that consistently chose (a), an increase in the number of individuals by level from Level 2 to 4 was observed in the Type 2 sentences, but this was not the case in the Type 4 sentences. Rather, in the Type 4 sentences, the percentages of individuals that consistently chose (a) were very similar across the levels (Level 2 , $64 \%$, Level 3, 67\%, Level 4, 67\%, Level 5, 67\%). The number of individuals that consistently chose (c) also increased in the Type 4 sentences but remained the same in Level (5).

I. Results of Type 2 and Type 4 Sentences Combined

Table VI lists the results for both the Type 2 and Type 4 sentences combined.

TABLE VI.

RESULTS OF TYPE 2 AND 4 SENTENCES COMBINED

\begin{tabular}{cccc}
\hline Level & Answer (a) & Answer (b) & Answer (c) \\
\hline L2 $(\mathrm{n}=11)$ & $57 / 88(64.8 \%)$ & $19 / 88(21.6 \%)$ & $12 / 88(13.6 \%)$ \\
L3 $(\mathrm{n}=12)$ & $61 / 96(63.5 \%)$ & $11 / 96(11.5 \%)$ & $24 / 96(25 \%)$ \\
L4 $(\mathrm{n}=9)$ & $51 / 72(70.8 \%)$ & $12 / 72(16.7 \%)$ & $9 / 72(12.5 \%)$ \\
L5 $(\mathrm{n}=9)$ & $35 / 72(48.6 \%)$ & $9 / 72(12.5 \%)$ & $28 / 72(38.9 \%)$ \\
Natives $(\mathrm{n}=20)$ & $117 / 160(73.1 \%)$ & $24 / 160(15 \%)$ & $19 / 160(11.9 \%)$ \\
\hline
\end{tabular}

Levels 2 to 4 showed a high preference for (a), but the Level 5 learners showed the least preference for (a) (48.6\%). Rather, they were more divided in their answers and showed the highest number of (c) answers (38.9\%). This is interesting because the native speaker group showed the highest preference for (a) with only $15 \%$ for (b) and $11.9 \%$ for (c). This means that in an overall comparison of sentences containing empty pronouns with quantified NP antecedents, 
the tendency was for the Level 5 learners to choose either that NP or an extra-sentential referent as the antecedent.

None of the individuals in any of the JFL groups or the Native speaker group chose all (a) responses for all of the Type 1 to 4 sentences. Only two learners in Level 2 chose (a) consistently in all of the Type 1 to 4 sentences. This suggests that most of the participants were discriminating in their choices across the different sentence types.

\section{DISCUSSION}

The overall results show that $2^{\text {nd }}$ year learners' judgments on sentences with overt pronouns and quantifier antecedents (i.e. Type 1 and Type 3), differ from those of the native speaker control group. The results from the sentences with empty pronouns and quantifier antecedents (i.e. Type 2 and Type 4) showed that although these sentences could be ambiguously interpreted since there was no discourse context, all groups showed a preference for choice (a). It is not clear why the native speakers did not show a high preference for option (c), although the combined Type 2 and Type 4 sentence percentages show that the Level 5 learners showed the highest preference for option (c).

\section{CONCLUSION}

In conclusion, the results show that $2^{\text {nd }}$ year Japanese learners treat the quantifiers as viable antecedents for the overt pronouns in the test sentences (i.e. disobeying the OPC), but that as the level of the learners increases, the number of correct rejections tends to increase, and the learners eventually acquire the OPC with more exposure to the language. This follows the findings of Masumoto (2008) and Pimentel and Nakayama (2012a) and offers further evidence against Kanno's $(1997,1998)$ results. The present study was limited in that it only examined learners' judgments of sentences containing overt and empty pronouns. Future research is needed to examine how JFL learners would apply empty and overt pronouns in a production task. Furthermore, from the perspectives of both comprehension and production, more studies are necessary to determine what possible input triggers there might be that account for JFL learners to eventually acquire knowledge of the correct distribution of overt and empty pronouns in Japanese.

\section{REFERENCES}

[1] Hoji, H. (1991). Demonstrative binding and principle B. NELS 25. 255-271. GSLA. University of Massachusetts, Amherst

[2] Kanno, K. (1997). The acquisition of null and overt pronominals in Japanese by English speakers. Second Language Research, 13.3, 265-287.

[3] Kanno, K. (1998). The stability of UG principles in second language acquisition: Evidence from Japanese. Linguistics, 36.6, $1125-1146$.

[4] Masumoto, A. (2008). Overt pronouns and bound variable reading in L2 Japanese. M.A. Thesis. The Ohio State University.

[5] Montalbetti, M. (1984). After binding: On the interpretation of pronouns. Doctoral dissertation. MIT.

[6] Pimentel, C. L. (2014). Pronominal interpretations in L2 Japanese. Doctoral dissertation. The Ohio State University.

[7] Pimentel, C.L. \& M. Nakayama. (2012a). Pronominal interpretations in L2 Japanese. Journal of Japanese Linguistics 28, 111131.

[8] Pimentel, C.L. \& M. Nakayama. (2012b). L2 Pronominal interpretations revisited. The 14th Annual Conference of the Japanese Society of Language Sciences Handbook, 155-156.

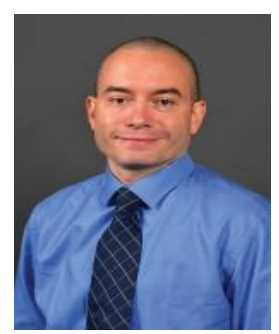

Carlos L. Pimentel received a B.A. in Japanese and linguistics from the University of MassachusettsAmherst (1995), an M.A. in Japanese literature from the University of Massachusetts-Amherst (2006), and a Ph.D. in East Asian Languages and Literatures with specialization in Japanese linguistics from The Ohio State University (2014). He served as the Director of the Japanese program at the University of Maryland for three years where he also taught Japanese language and linguistics. He is currently an Assistant Professor of Japanese language and linguistics at Western Michigan University in Kalamazoo. 